\title{
Anatomical and clinical predictors of valve dysfunction and aortic dilation in bicuspid aortic valve disease
}

\author{
Arturo Evangelista, ${ }_{1}$ Pastora Gallego, ${ }^{2}$ Francisco Calvo-Iglesias, ${ }^{3}$ Javier Bermejo, ${ }^{4}$ \\ Juan Robledo-Carmona, ${ }^{5}$ Violeta Sánchez, ${ }^{6}$ Daniel Saura, ${ }^{7}$ Roman Arnold, ${ }^{8}$ \\ Amelia Carro, ${ }^{1}$ Giuliana Maldonado, ${ }^{1}$ Augusto Sao-Avilés, ${ }^{1}$ Gisela Teixidó, 1 \\ Laura Galian, ${ }^{1}$ José Rodríguez-Palomares, ${ }^{1}$ David García-Dorado ${ }^{1}$
}

\section{- Additional material is published online only. To view please visit the journal online (http://dx.doi.org/10.1136/ heartjnl-2017-311560). \\ For numbered affiliations see end of article. \\ Correspondence to \\ Dr Arturo Evangelista, Servei de Cardiologia, Hospital Universitari Vall d'Hebron, Po Vall d'Hebron 119, Barcelona 08036, Spain; arturevangelistam asip@gmail.com}

Received 21 March 2017 Revised 3 July 2017

Accepted 11 July 2017 Published Online First 1 September 2017

- http://dx.doi.org/10.1136/ heartjnl-2017-312133

\section{Check for updates}

To cite: Evangelista $\mathrm{A}$, Gallego P, Calvo-

Iglesias F, et al. Heart 2018;104:566-573.

\section{ABSTRACT}

Objective Bicuspid aortic valve (BAV) is associated with early valvular dysfunction and proximal aorta dilation with high heterogeneity. This study aimed to assess the determinants of these complications.

Methods Eight hundred and fifty-two consecutive adults diagnosed of BAV referred from cardiac outpatient clinics to eight echocardiographic laboratories of tertiary hospitals were prospectively recruited. Exclusion criteria were aortic coarctation, other congenital disorders or intervention. BAV morphotype, significant valve dysfunction and aorta dilation ( $\geq 2 \mathrm{Z}$-score) at sinuses and ascending aorta were established.

Results Three BAV morphotypes were identified: right-left coronary cusp fusion $(\mathrm{RL})$ in $72.9 \%$, right-noncoronary (RN) in $24.1 \%$ and left-non-coronary (LN) in $3.0 \%$. BAV without raphe was observed in $18.3 \%$. Multivariate analysis showed aortic regurgitation (23\%) to be related to male sex (OR: $2.80, p<0.0001)$ and valve prolapse (OR: $5.16, p<0.0001)$, and aortic stenosis (22\%) to BAV-RN (OR: 2.09, $\mathrm{p}<0.001)$, the presence of raphe (OR: $2.75, p<0.001)$, age (OR: 1.03; $p<0.001)$, dyslipidaemia (OR: 1.77, $\mathrm{p}<0.01)$ and smoking (OR: $1.63, p<0.05)$. Ascending aorta was dilated in $76 \%$ without differences among morphotypes and associated with significant valvular dysfunction. By contrast, aortic root was dilated in 34\% and related to male sex and aortic regurgitation but was less frequent in aortic stenosis and BAV-RN.

Conclusions Normofunctional valves are more prevalent in BAV without raphe. Aortic stenosis is more frequent in BAV-RN and associated with some cardiovascular risk factors, whereas aortic regurgitation $(A R)$ is associated with male sex and sigmoid prolapse. Although ascending aorta is the most commonly dilated segment, aortic root dilation is present in one-third of patients and associated with AR. Remarkably, BAV-RL increases the risk for dilation of the proximal aorta, whereas BAV-RN spares this area.

\section{INTRODUCTION}

Bicuspid aortic valve (BAV) is the most common congenital heart abnormalities. This condition is associated with significant valvular disease including aortic valve stenosis, regurgitation and infective endocarditis and with many vascular abnormalities such as ascending aorta dilation or coarctation of the aorta. ${ }^{1-4}$ The prevalence of ascending aorta dilation among patients with BAV ranges from $20 \%$ to $84 \%$ depending on the study population. ${ }^{5-8}$ This aortopathy bears an increased risk of life-threatening complications such as rupture and dissection. ${ }^{1-3}$ Community studies showed that, 20 years after diagnosis, aortic valve surgery or some type of cardiovascular surgery was required in approximately $25 \%$ of patients. ${ }^{78}$ Although most BAV are predisposed to progressive and accelerated calcification, little is known on variables related to significant valvular dysfunction. ${ }^{9}$ Similarly, several studies reported significant disparities in the relationship between valve characteristics and ascending aorta dilation. ${ }^{10-14}$ Discordant results may be due to the retrospective design of studies, small series with different study population characteristics and the use of different measurement methods or normal size values.

The aim of the present study was to assess the main determinants of valvular dysfunction and aorta dilation in a large series of patients with BAV.

\section{METHODS}

\section{Study population}

Nine hundred and ten consecutive adults (age $\geq 18$ years) diagnosed of BAV referred from cardiac outpatient clinics to echocardiographic laboratories between 2012 and 2015 were prospectively recruited from eight tertiary hospitals. Patients were not included if there was evidence of previous aortic valvuloplasty, corrective aortic surgery, aortic valve endocarditis, aortic coarctation or other congenital aortic diseases. Fifty-six of the 908 patients were excluded since a definitive diagnosis of BAV could not be established owing to suboptimal image quality. The study population comprised 852 patients. This prospective study was approved by the institutional review board of each hospital.

\section{Echocardiography}

Echocardiographic studies were performed with harmonic imaging at all institutions. Complete echocardiographic studies were conducted by expert echocardiographers at each centre following a predefined protocol. Images were stored in DVD 
and sent to a core lab (Hospital Vall d'Hebron) where two expert echocardiographers analysed and measured all the studies. Doubtful images were discussed and diagnosed by consensus. BAV was defined as the presence of two cusps and commissures, with or without raphe in either structure. Each aortic valve was analysed and characterised in systole and diastole. Diagnosis was established by the systolic fish-mouth appearance of the orifice in parasternal short axis views using a zoom tool. Valve morphotype was categorised as right and left (RL) coronary cusps fusion (anteroposterior BAV), the right coronary and non-coronary (RN) cusps fusion (right-left BAV) and the left coronary and non-coronary (LN) cusps fusion. Comprehensive echo Doppler examination was performed to assess valvular dysfunction severity. Based on current guidelines, valvular dysfunction was defined as none, mild, moderate or severe aortic stenosis (AS) or aortic regurgitation (AR). Patients with mixed BAV disease were classified according to the predominant functional valve lesion. Patients were categorised according to the degree and type of valvular dysfunction in a non-significant group (normally functioning, mild AS or mild AR), an AS-dominant group (patients with moderate or severe AS) and an AR-dominant group (patients with moderate or severe AR). The degree of valvular calcification or sigmoid prolapse was established and reported in all cases.

Aortic coarctation was ruled out by assessing abdominal aorta flow in long axis view (continuous flow pattern) and proximal descending aorta flow by colour and continuous Doppler by suprasternal view. Calcified aortic valve was considered when more than mild and localised calcification was visualised according to the Yousry classification. ${ }^{15}$

The ascending aorta was measured by two-dimensional echocardiography using the parasternal long axis view. Further cephalad segments of the ascending aorta were imaged by moving the transducer up 1 or two interspaces and the aortic arch via suprasternal notch. Aortic diameter was measured at the annulus, root (maximum dilation of Valsalva sinuses), sinotubular junction (STJ) and ascending aorta at the level of maximum ascending aorta diameter; the proximal aortic arch was measured via suprasternal or supraclavicular window. Measurements of the aortic root, STJ, ascending aorta and arch were taken using the leading edge to leading edge convention in end-diastole (online supplementary figure 1). The aortic annulus was measured by the inner edge to inner edge convention at end-systole. Normal aorta size was defined by the reference values reported for the aortic root and ascending aorta based on established guidelines, considering age, body size and sex. ${ }^{16}$ Significant aorta dilation was considered when aorta diameter was $>45 \mathrm{~mm}$ given that this diameter can generate clinical decision making in patients with surgical indication.

The aorta phenotype classification included in this study was assigned according to the segment of the vessel with the largest diameters: 'ascending aorta' type, if the diameter distal to the STJ exceeded that at the root, and 'root' type if the maximum diameter observed was at the level of the sinuses. ${ }^{17}$

\section{Statistical analysis}

Continuous demographic variables were expressed as mean \pm SD. The Kolmogorov-Smirnov test was used to evaluate the normal distribution of variables. Intergroup differences for continuous parameters were assessed by Student's t-test if they presented a normal distribution or analysis of variance with Bonferroni correction for multiple comparisons, and Mann-Whitney U test if they did not present a normal distribution. For categorical variables, general characteristics of the sample were assessed by percentages $\left(\chi^{2}\right.$ test). Multivariate linear regression analysis with a forward selection procedure was used to identify independent variables associated with aortic root or ascending aorta dilation; however, multivariate logistic regression analysis was made to identify factors associated with valvular dysfunction. Variables were entered in the model if $\mathrm{p}<0.25$ on univariate analysis.

Definitive BAV cases in which morphotype classification or the presence of raphe were not definitively established were included in the study but excluded from univariate or multivariate analyses. A two-tailed $\mathrm{p}$ value $<0.05$ was considered statistically significant. SPSS V.19.0 software version was used for the analysis.

\section{RESULTS}

\section{Patient characteristics and BAV phenotypes}

Characteristics of the 852 patients (mean age: $47.4 \pm 16.8$ years; $70.2 \%$ male) enrolled in our cross-sectional multicentre study are shown in table 1. BAV-RL was significantly more frequent (72.9\%) than BAV-RN (24.1\%) and BAV-LN (3.0\%). Pure BAV without raphe was observed in $18.3 \%$ of cases. BAV morphotype was not definitively established in 43 patients (5\%) nor the presence of raphe in $64(7.5 \%)$. No differences in demographic variables or the presence of raphe among valvular morphotypes were observed.

\section{Factors associated with valvular dysfunction}

Sigmoid prolapse was diagnosed in $110(12.9 \%)$ patients and significant valve calcification in $210(24.6 \%)$. Compared with BAV-RL, BAV-RN more frequently showed valve prolapse $(22.1 \%$ vs $10.5 \% ; \mathrm{p}<0.0001)$ and valve calcification $(31.8 \%$ vs $23.2 \% \mathrm{p}<0.01$ ) (table 1 ). Several demographic variables were related to valve calcification: age $(56.1 \pm 13.2$ years vs $44.4 \pm 16.9 ; \mathrm{p}<0.001)$, arterial hypertension $(38.4 \%$ vs $21.1 \%$; $\mathrm{p}<0.001)$, dyslipidaemia $(45.9 \%$ vs $20.8 \%, \mathrm{p}<0.001)$, diabetes $(52.3 \%$ vs $25.3 \%, \mathrm{p}<0.001)$ and smoking $(45.1 \%$ vs $25.9 \%$, $\mathrm{p}<0.001)$. Calcification was also more frequent in BAV with raphe $(28.9 \%$ vs $16.9 \%, p=0.002)$ and BAV-RN and BAV-LN than in BAV-RL $(\mathrm{p}=0.02)$.

Absence of significant valvular dysfunction was established in 467 (54.8\%) patients, significant AR in 201 (23.6\%), 56 of them severe $(27.8 \%)$, and significant AS in 184 (21.6\%), 75 of them severe $(40.8 \%)$ (online supplementary table 1 ). The relationship between valvular dysfunction and demographic and valve characteristics is shown in table 2. On multivariate analysis, variables independently related to AS were age, dyslipidaemia, smoking, presence of raphe and BAV-RN, and those related to AR were male sex and the presence of sigmoid prolapse. AR was more prevalent in men $(28.3 \%$ vs $12.6 \%$; $\mathrm{p}<0.001)$ (online supplementary table 2 ). When valvular dysfunction by age was considered, AR was more prevalent in young patients and AS in older patients (figure 1). Patients with valve raphe presented AS more frequently than patients without raphe $(25.8 \%$ vs $11.1 \%$, $\mathrm{p}<0.001$ ) (table 3).

\section{Variables related to aortic dilation and aorta phenotype}

Aorta dilation was diagnosed in 663 patients $(76.1 \%)$, aortic root in $282(33.9 \%)$ and/or ascending aorta in $632(76.1 \%)$. In only 21 cases $(2.5 \%)$ could aortic diameters not be measured. No differences were found between BAV morphotypes and ascending aorta diameter. However, BAV-RN showed a smaller aortic root diameter than BAV-RL $(34.3 \pm 5.3 \mathrm{~mm}$ vs $37.5 \pm 5.6 \mathrm{~mm} ; \mathrm{p}<0.01)$ and larger arch diameter $(33.3 \pm 6.3 \mathrm{~mm}$ vs $30.9 \pm 5.1 \mathrm{~mm}$; 
Table 1 Baseline characteristics of the study participants according to BAV morphotype

\begin{tabular}{|c|c|c|c|c|c|}
\hline Variable & $\begin{array}{l}\text { All patients } \\
\mathrm{n}=852\end{array}$ & $\begin{array}{l}\text { BAV-RL } \\
n=590 \\
(72.9 \%)\end{array}$ & $\begin{array}{l}\text { BAV-RN } \\
n=195 \\
(24.1 \%)\end{array}$ & $\begin{array}{l}\text { BAV-LN } \\
n=24 \\
(3.0 \%)\end{array}$ & $\mathrm{p}$ Value \\
\hline \multicolumn{6}{|l|}{ Demographics and clinical data } \\
\hline Age, years & $47.4 \pm 16.8$ & $47.6 \pm 17.1$ & $46.4 \pm 16.1$ & $49.6 \pm 15.6$ & 0.425 \\
\hline Male, $n(\%)$ & $598(70.2)$ & $425(72.0)$ & $132(67.7)$ & $16(66.6)$ & 0.423 \\
\hline $\mathrm{BSA}, \mathrm{kg} / \mathrm{m}^{2}$ & $1.85 \pm 0.2$ & $1.86 \pm 0.1$ & $1.83 \pm 0.2$ & $1.85 \pm 0.2$ & 0.075 \\
\hline Smoking, $n(\%)$ & $239(28.1)$ & $167(28.3)$ & $65(33.3)$ & $5(20.8)$ & 0.235 \\
\hline Hypertension, $n(\%)$ & $260(30.1)$ & $194(32.9)$ & $56(28.7)$ & $8(33.3)$ & 0.201 \\
\hline Diabetes mellitus, $n(\%)$ & $44(5.2)$ & $27(4.6)$ & $14(7.2)$ & $2(8.3)$ & 0.196 \\
\hline Dyslipidaemia, $n(\%)$ & $185(21.7)$ & $132(22.4)$ & $43(22.1)$ & $8(33.3)$ & 0.921 \\
\hline \multicolumn{6}{|l|}{ Valve abnormality and dysfunction } \\
\hline Raphe, $n(\%)$ & $644(75.6)$ & $467(79.2)$ & $146(74.9)$ & $20(83.3)$ & 0.066 \\
\hline Sigmoid prolapse, $n(\%)$ & $110(12.9)$ & $62(10.5)$ & $43(22.1)^{*}$ & $4(16.7)$ & 0.0001 \\
\hline Valve calcification, $n(\%)$ & $210(24.6)$ & $137(23.2)$ & $62(31.8)^{* * *}$ & $9(37.5)$ & 0.022 \\
\hline Normofunctional, $n(\%)$ & $467(54.8)$ & $327(55.4)^{* * *}$ & $93(47.7)$ & $12(50.0)$ & 0.015 \\
\hline $\mathrm{AS}, n(\%)$ & $184(21.6)$ & $117(19.8)$ & $58(29.7)^{* *}$ & $6(25.0)$ & 0.004 \\
\hline $\mathrm{AR}, n(\%)$ & $201(23.6)$ & $146(24.8)$ & $44(22.6)$ & $6(25.0)$ & 0.534 \\
\hline \multicolumn{6}{|l|}{ Aortic diameter, dilation and morphotype } \\
\hline Aortic annulus, mm & $23.3 \pm 3.3$ & $23.4 \pm 3.3$ & $23.1 \pm 3.2$ & $22.5 \pm 4.3$ & 0.246 \\
\hline Aortic root, mm & $36.7 \pm 5.7$ & $37.5 \pm 5.6^{*}$ & $34.3 \pm 5.3$ & $38.9 \pm 6.8$ & 0.0001 \\
\hline STJ, mm & $30.5 \pm 6.4$ & $30.9 \pm 6.4^{* *}$ & $29.0 \pm 6.1$ & $31.4 \pm 8.8$ & 0.0001 \\
\hline Ascending aorta, mm & $40.6 \pm 7.6$ & $41.2 \pm 7.6$ & $39.9 \pm 7.4$ & $40.1 \pm 7.5$ & 0.052 \\
\hline Aortic arch, mm & $31.6 \pm 5.5$ & $30.9 \pm 5.1$ & $33.3 \pm 6.3^{*}$ & $33.9 \pm 5.9$ & 0.0001 \\
\hline Aortic dilation & $663(79.8)$ & $486(83.1)^{* *}$ & $145(74.4)$ & $19(79.2)$ & 0.037 \\
\hline Root dilation phenotype & $122(14.7)$ & $101(17.1)^{*}$ & $10(5.1)$ & $7(29.2)$ & 0.001 \\
\hline Ascending aorta dilation phenotype & $541(65.1)$ & $385(65.3)$ & $135(69.2)$ & $12(50)$ & 0.131 \\
\hline
\end{tabular}

${ }^{*}$ BAV-RL versus BAV-RN: ${ }^{*} p<0.001 ;{ }^{* *} p<0.01 ;{ }^{* * *} p<0.05$.

$A R$, aortic regurgitation; $A S$, aortic stenosis; BAV, bicuspid aortic valve

BSA, body surface area; LN, left coronary and non-coronary; RL, right and left; RN, right coronary and non-coronary; STJ, sinotubular junction.

$\mathrm{p}<0.01)$. By contrast, BAV-LN showed similar aortic root diameters to BAV-RL $(38.9 \pm 6.8 \mathrm{~mm})$ and aortic arch diameters to BAV-RN $(33.9 \pm 5.9 \mathrm{~mm}$ ) (table 1$)$. Aortic root and maximum ascending aorta diameters by age in BAV-RL versus BAV-RN are shown in figure 2. Maximum aortic root diameter was larger in patients with AR compared with AS or non-significant dysfunction $(39.0 \pm 5.9 \mathrm{~mm}, 36.1 \pm 5.4 \mathrm{~mm}, 35.8 \pm 5.5 \mathrm{~mm}$, respectively; $\mathrm{p}<0.0001)$, and maximum ascending aorta in AS compared with the remaining groups $(43.7 \pm 7.4 \mathrm{~mm}, 41.5 \pm 7.1 \mathrm{~mm}$ and $38.9 \pm 7.5 \mathrm{~mm} ; \mathrm{p}<0.0001$ ) (online supplementary table 1 ). Univariate and multivariate analyses among aortic root and ascending aorta dilation and demographic, valve characteristics and valvular dysfunction are shown in (online supplementary table 3). In patients with aorta dilation, aortic root phenotype was observed in $122(18.4 \%)$ and ascending aorta phenotype in $541(81.6 \%)$. Aortic root phenotype was independently associated with younger age, male sex and AR and less frequently associated with BAV-II and AS, and ascending aorta phenotype with age, female sex and AS (table 4). Distribution of aorta dilation phenotypes in relation to valvular morphotype and valvular dysfunction is shown in figure 3 . In patients without aortic dilation, ascending aorta phenotype was also present in 91 (54.2\%) of 168 patients, being more frequent in BAV-RN than in BAV-RL (73.4\% vs $45.5 \%$, respectively; $\mathrm{p}<0.01)$.

Significant aorta dilation $(>45 \mathrm{~mm})$ was observed in 261 (31.3\%) patients, 66 (7.9\%) was in aortic root and/or 212 $(25.5 \%)$ in ascending aorta. In $79(9.3 \%)$ patients, aorta dilation was $>50 \mathrm{~mm}$. In the multivariate analysis, variables related to significant aortic root dilation were age, male sex and AR, with
BAV-RN being less frequent, and variables related to significant ascending aorta dilation were age, AR and AS (online supplementary table 4).

\section{DISCUSSION}

This large prospective study of an adult BAV population found significant heterogeneity in valvular dysfunction and aorta dilation depending on valve characteristics and several risk factors. AR was associated with male sex and valve prolapse and AS with several cardiovascular risk factors, presence of raphe and BAV-RN morphotype. Ascending aorta dilation was common, $71 \%$ in normofunctional and $86 \%$ in dysfunctional valve, with no differences among valve morphotypes. By contrast, aortic root dilation was present in only $34 \%$ of patients and strongly associated with AR. BAV-RN morphotype spared aortic root dilation but had significant arch enlargement compared with BAV-RL. Characteristically, patients with aortic root dilation phenotype were younger, more likely to be male, had increased frequency of AR but low frequency of AS and BAV-RN morphotype.

\section{Valvular dysfunction}

BAV is prone to premature fibrosis, stiffening and calcium deposition. Stenotic calcification of a BAV is accelerated and may appear one decade earlier than that of a tricuspid aortic valve. ${ }^{9}$ In the present study, moderate to severe valve calcification was identified in $27 \%$ of cases and related to conventional risk factors: age, arterial hypertension, dyslipidaemia, diabetes and smoking. Although few studies analysed the predictors of 


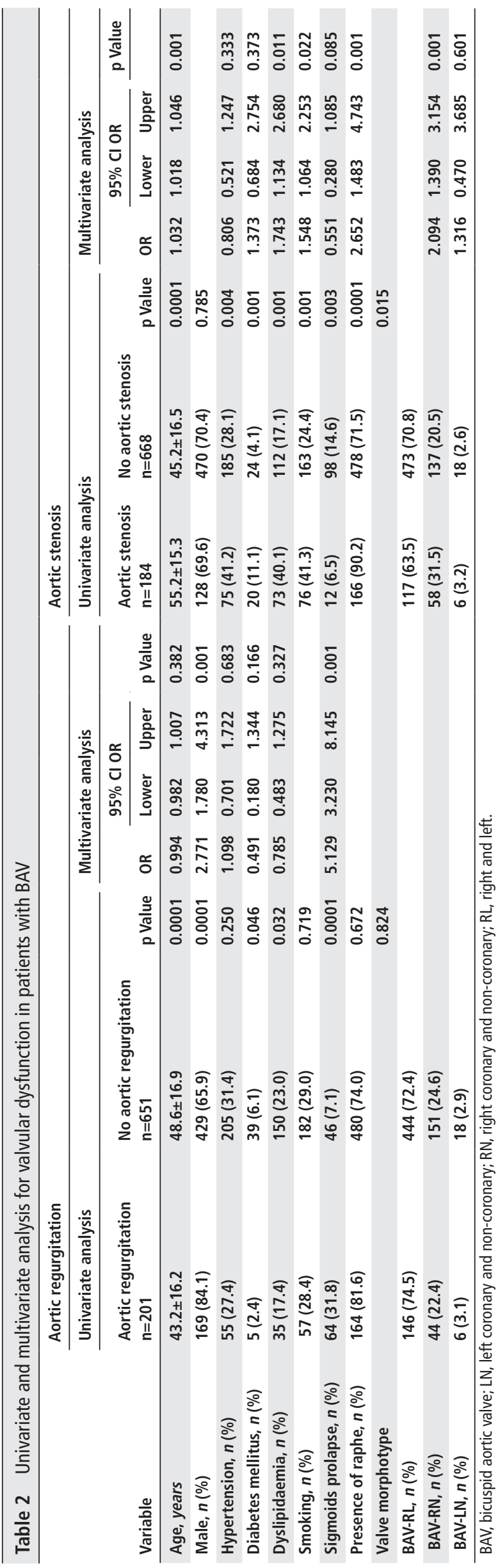

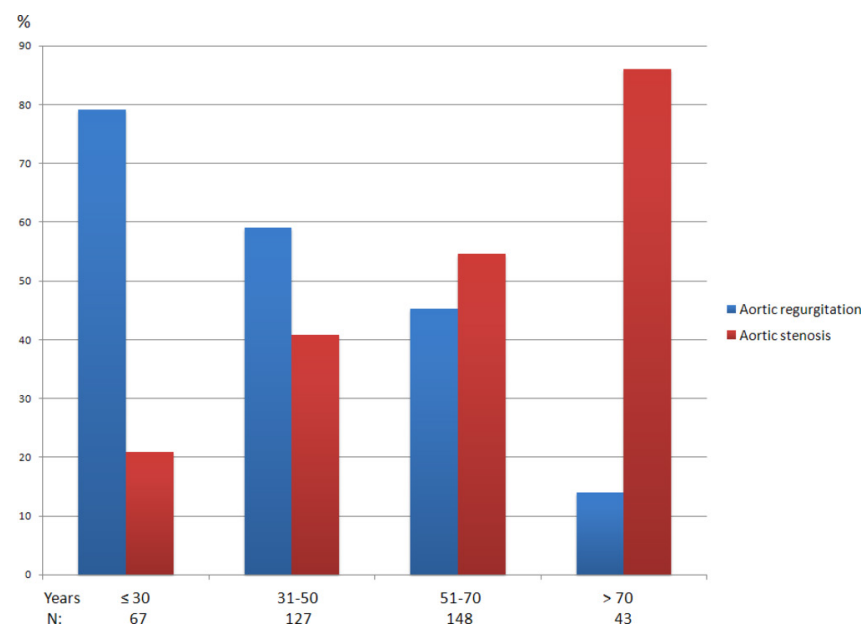

Figure 1 Prevalence of valvular dysfunction according to age. When valvular dysfunction by age was considered, AR was more prevalent in young patients and AS in older patients. AR, aortic regurgitation; AS, aortic stenosis.

valve degeneration in BAV, Michelena et $a l^{7}$ in a community study, showed age, dyslipidaemia and the presence of raphe to be associated with valve degeneration. Based on these findings, a control of cardiovascular risk factors and some pharmacological treatment such as statin therapy could be beneficial and reduce or delay the degeneration of the BAV. ${ }^{18}$

In addition to the known male predominance in BAV, we, like others, found a higher prevalence of AR in $\operatorname{men}^{141719}$ and normofunctional valves in women, with a similar prevalence of AS in both sexes. While a clear explanation for this sex difference is lacking, it has also been reported in some surgical series, ${ }^{20}$

Table 3 Patient's characteristics, valve dysfunction, aortic diameters and dilation according to the presence of raphe

\begin{tabular}{|c|c|c|c|}
\hline Variable & $\begin{array}{l}\text { No raphe } \\
\mathrm{n}=144 \\
(18.8 \%)\end{array}$ & $\begin{array}{l}\text { Raphe } \\
n=644 \\
(81.2 \%)\end{array}$ & p Value \\
\hline \multicolumn{4}{|l|}{ Demographics and clinical data } \\
\hline Age, years & $46.2 \pm 17.2$ & $47.6 \pm 16.7$ & 0.32 \\
\hline Female, $n(\%)$ & $93(65)$ & $465(72)$ & 0.08 \\
\hline $\mathrm{BSA}, \mathrm{kg} / \mathrm{m}^{2}$ & $1.83 \pm 0.22$ & $1.85 \pm 0.19$ & 0.50 \\
\hline Smoker, $n(\%)$ & 16 (11.8) & $91(14.4)$ & 0.496 \\
\hline Hypertension, $n(\%)$ & $50(36.5)$ & $201(31.5)$ & 0.269 \\
\hline \multicolumn{4}{|l|}{ Valve dysfunction } \\
\hline Non-significant, $n$ (\%) & $94(65.3)$ & $314(48.7)$ & 0.001 \\
\hline AS dominant, $n(\%)$ & $16(11.1)$ & $166(25.8)$ & \\
\hline AR dominant, $n(\%)$ & $34(23.6)$ & $164(25.4)$ & \\
\hline \multicolumn{4}{|l|}{ Aortic diameter } \\
\hline Aortic annulus, mm & $23.6 \pm 3.3$ & $23.2 \pm 3.3$ & 0.174 \\
\hline Aortic root, mm & $37.1 \pm 6.3$ & $36.8 \pm 5.6$ & 0.515 \\
\hline STJ, mm & $31.1 \pm 6.0$ & $30.4 \pm 6.5$ & 0.071 \\
\hline Ascending aorta, mm & $40.6 \pm 7.1$ & $41.1 \pm 7.6$ & 0.652 \\
\hline Aortic arch, mm & $30.1 \pm 5.55$ & $31.7 \pm 5.5$ & 0.238 \\
\hline \multicolumn{4}{|l|}{ Aortic dilation } \\
\hline Non-dilation, $n(\%)$ & $22(15.3)$ & $121(18.8)$ & 0.341 \\
\hline Root dilation, $n(\%)$ & $60(41.7)$ & $214(33.2)$ & 0.066 \\
\hline Ascending aorta dilation, $n(\%)$ & $116(81.1)$ & $498(77.6)$ & 0.373 \\
\hline
\end{tabular}

$A R$, aortic regurgitation; $A S$, aortic stenosis; $B S A$, body surface area; STJ, sinotubular junction. 


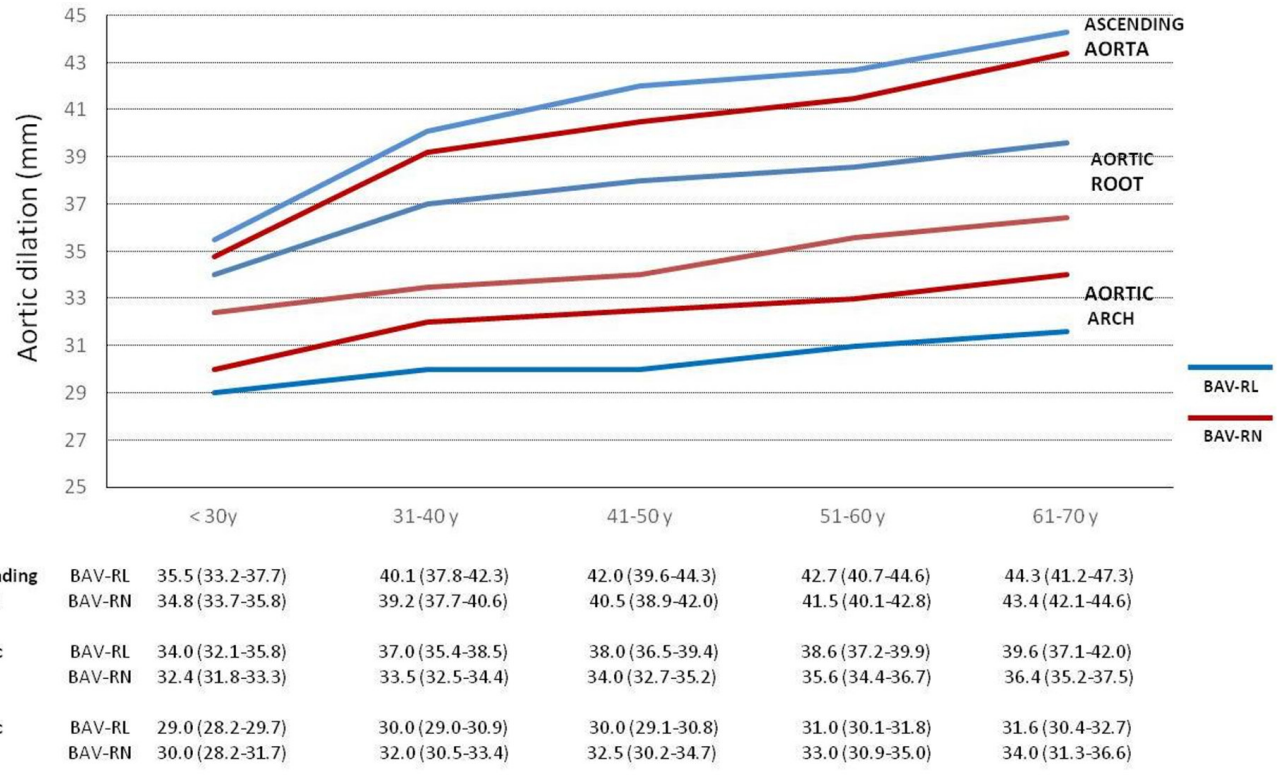

Figure 2 Aortic diameters according to age. Graph representing mean aortic diameters at the level of the aortic root, ascending aorta and aortic arch in patients with BAV-RL and BAV-RN. Note the homogeneous enlargement of the diameters over time in the three aorta segments. However, while ascending aorta diameters were similar between BAV types, aortic root diameters were consistently larger in BAV-RL and aortic arch diameters in patients with BAV-RN. Table shows mean and Cls of aortic diameters according to age at the three different aorta levels. BAV, bicuspid aortic valve; $\mathrm{RL}$, right and left; RN, right coronary and non-coronary.

The relationship between BAV phenotype and valvular dysfunction has been described, with controversial results. In our series, BAV-RN was found to be associated more often with valve calcification and AS. Although clinical research based on a presurgical cohort found no such correlation, ${ }^{10-12}$ an association between BAV-RN and AS was reported in other series. Pure BAV without raphe was diagnosed in around $20 \%$ of cases and showed a lower prevalence of valvular dysfunction. ${ }^{11-13}$ Similar results were reported in a recent large retrospective study; however, in that series, patients with raphe were older and with higher risk factor prevalence. ${ }^{21}$ The main location of calcification is often in the raphe, which may be a major factor leading to valve degeneration and AS. However, ${ }^{3,17}$ the most common mechanisms leading to chronic regurgitation in BAV, acting either alone or in combination, are annular dilation and cusp prolapse or retraction. In our series, AR was more frequent in male sex and associated with sigmoid prolapse.

\section{Aorta dilation and related factors}

Aorta dilation is a common finding in patients with BAV. Several studies reported a prevalence ranging from $16 \%$ to $78 \%$ at the sinus of Valsalva and 33\%-68\% at the ascending aorta. ${ }^{1-35611} 1417$ In the present study, the aortic root was dilated in $34 \%$ of cases and ascending aorta in 76\%, and ascending aorta dilation did not differ between BAV-RL and BAV-RN morphotypes. Controversial data exist regarding the relationship between BAV morphology and aorta dilation due to different study populations, measurement methods or the threshold used to define dilation in each study. ${ }^{10-12} 1722$ However, BAV-RN spared aortic root dilation (18\% vs 39\%) and presented a larger aortic arch size than BAV-RL. Patients with BAV-LN, a morphotype not included in most studies, had similar aortic root dilation to those with BAV-RL and arch diameter to those with BAV-RN.
Concurring with other series, AR was independently associated with aortic root dilation ${ }^{5} 822$ and both AR and AS with ascending aorta dilation. ${ }^{458}$ We found males had a higher prevalence of root phenotype. This root phenotype was also associated with younger age but with the presence of AR and absence of AS. This infrequent aorta phenotype has been proposed as the form of bicuspid aortopathy most likely to be associated with a genetic cause and may represent a higher risk condition. ${ }^{11}{ }^{17} \mathrm{~A}$ recent meta-analysis demonstrated a 10-fold higher risk of aortic dissection in BAV patients who undergo isolated AVR for AR compared with AS. ${ }^{23}$

In our series, $30 \%$ of patients with normofunctional valve had aortic root dilation and $70 \%$ ascending aorta dilation. Dilation of the tubular part, even with a normally functioning valve, was also considered a strong argument in favour of the existence of genetically determined forms of aortopathy. ${ }^{2-5}{ }^{24}$ However, Robicsek et $a l^{25}$ found the clinically normal BAV to be in fact morphologically stenotic and producing eccentric turbulent transvalvular flow. Girdauskas et $a^{26}$ showed comparable correlation patterns between functional aortic root parameters and severity/expression of aortopathy in patients with bicuspid versus tricuspid aortic valve stenosis, thereby supporting the fact that aortopathy may be the haemodynamically triggered phenomenon. Recent four-dimensional flow MRI studies showed different helical flow in the aortic root and ascending aorta depending on the valvular morphotype of normofunctional $\mathrm{BAV}^{27}{ }^{28}$; these eccentric jets lead to a differential distribution of aortic wall shear stress and subsequent vascular remodelling of the aortic wall. ${ }^{23}$ We found significant aortic root dilation $(>45 \mathrm{~mm})$ to be related to age, male sex and significant AR, whereas ascending aorta dilation ( $>45 \mathrm{~mm}$ ) was related to age and significant AR and AS, thereby suggesting that haemodynamic abnormalities secondary to valvular dysfunction may produce an additional effect on aorta dilation. $^{29}$ 
Table 4 Demographic and valve characteristics of BAV patients according to aortic dilation and aorta phenotypes. Univariate and multivariate analysis

\begin{tabular}{|c|c|c|c|c|c|c|}
\hline \multirow[b]{2}{*}{ Aorta phenotype } & \multicolumn{3}{|c|}{$\begin{array}{l}\text { Non-dilated aorta } \\
168(20.2 \%)\end{array}$} & \multicolumn{3}{|l|}{$\begin{array}{l}\text { Dilated aorta } \\
663(79.8 \%)\end{array}$} \\
\hline & $\begin{array}{l}\text { Aortic root } \\
77(45.8 \%)\end{array}$ & $\begin{array}{l}\text { Ascending aorta } \\
91(54.2 \%)\end{array}$ & $\mathrm{p}$ Value & $\begin{array}{l}\text { Aortic root } \\
122(18.4 \%)\end{array}$ & $\begin{array}{l}\text { Ascending aorta } \\
541(81.6 \%)\end{array}$ & $\mathrm{p}$ Value \\
\hline \multicolumn{7}{|l|}{$\begin{array}{l}\text { Univariate analysis } \\
\text { Demographics and clinical data }\end{array}$} \\
\hline Age, years & $42.1 \pm 17.4$ & $49.2 \pm 14.5$ & 0.066 & $41.5 \pm 16.9$ & $49.2 \pm 16.6$ & 0.0001 \\
\hline Male, $n(\%)$ & $58(75.3)$ & $61(67.0)$ & 0.307 & $106(86.9)$ & $363(67.1)$ & 0.0001 \\
\hline Smoking, $n(\%)$ & $15(19.4)$ & $28(31.0)$ & 0.139 & $32(26.4)$ & $163(30.1)$ & 0.502 \\
\hline Hypertension, $n(\%)$ & $15(19.4)$ & $27(27.2)$ & 0.099 & $32(26.4)$ & $185(34.2)$ & 0.009 \\
\hline Diabetes mellitus, $n(\%)$ & $3(4.8)$ & $10(11.1)$ & 0.143 & $4(3.3)$ & $27(5.2)$ & 0.511 \\
\hline Dyslipidaemia, $n(\%)$ & $13(17.3)$ & $22(24.1)$ & 0.332 & $20(16.5)$ & $130(24.0)$ & 0.074 \\
\hline \multicolumn{7}{|l|}{ Valve morphotype } \\
\hline & & & 0.006 & & & 0.001 \\
\hline BAV-RL, $n(\%)$ & $54(77.1)$ & 45 (49.4) & & $101(83.0)$ & $385(71.1)$ & \\
\hline BAV-RN, $n(\%)$ & $13(18.6)$ & $36(40.1)$ & & $10(8.2)$ & $135(25.1)$ & \\
\hline BAV-LN, $n(\%)$ & $3(4.3)$ & $2(2.2)$ & & $7(5.7)$ & $12(2.2)$ & \\
\hline \multicolumn{7}{|l|}{ Valve abnormality and function } \\
\hline Raphe, $n(\%)$ & $57(74.0)$ & $64(70.3)$ & 0.486 & $92(75.4)$ & $429(79.3)$ & 0.362 \\
\hline Sigmoid prolapse, $n(\%)$ & $7(9.1)$ & $9(9.9)$ & 0.999 & $23(18.9)$ & $71(13.1)$ & 0.112 \\
\hline Non-significant valve dysfunction, $n(\%)$ & $58(75.3)$ & $58(63.7)$ & 0.105 & $65(53.3)$ & $266(49.2)$ & 0.001 \\
\hline AS dominant, $n(\%)$ & $6(7.8)$ & $23(25.3)$ & & $7(5.7)$ & $148(27.4)$ & \\
\hline AR dominant, $n(\%)$ & $13(16.9)$ & $10(11.0)$ & & $50(41.0)$ & $127(23.5)$ & \\
\hline \multicolumn{7}{|c|}{ Multivariate analysis of aorta dilation phenotype } \\
\hline Aortic root phenotype & OR & & $95 \% \mathrm{Cl}$ & & $\mathrm{p}$ Value & \\
\hline Age & 0.97 & & $0.96-0.99$ & & 0.004 & \\
\hline Male & 3.98 & & $2.07-7.63$ & & 0.001 & \\
\hline Hypertension & 1.05 & & $0.59-1.88$ & & 0.846 & \\
\hline Dyslipidaemia & 1.01 & & $0.53-1.91$ & & 0.963 & \\
\hline Sigmoid prolapse & 1.07 & & $0.55-2.11$ & & 0.826 & \\
\hline BAV-RN & 0.28 & & $0.13-0.59$ & & 0.001 & \\
\hline BAV-LN & 2.79 & & $0.93-8.38$ & & 0.067 & \\
\hline AS dominant & 0.23 & & $0.10-0.54$ & & 0.001 & \\
\hline AR dominant & 1.27 & & $0.76-2.13$ & & 0.353 & \\
\hline \multicolumn{7}{|l|}{ Ascending aorta phenotype } \\
\hline Age & 1.02 & & $1.00-1.04$ & & 0.004 & \\
\hline Male & 0.25 & & $0.13-0.48$ & & 0.001 & \\
\hline Hypertension & 0.944 & & $0.53-1.68$ & & 0.846 & \\
\hline Dyslipidaemia & 0.985 & & $0.52-1.85$ & & 0.963 & \\
\hline Sigmoid prolapse & 0.92 & & $0.47-1.18$ & & 0.826 & \\
\hline BAV-RN & 3.55 & & $1.68-7.50$ & & 0.001 & \\
\hline BAV-LN & 0.35 & & $0.11-1.07$ & & 0.067 & \\
\hline AS dominant & 4.28 & & $1.84-9.94$ & & 0.001 & \\
\hline AR dominant, $n(\%)$ & 0.78 & & $0.46-1.31$ & & 0.353 & \\
\hline
\end{tabular}

$\mathrm{AS}$, aortic stenosis; $A R$, aortic regurgitation; BAV, bicuspid aortic valve; LN, left coronary and non-coronary; RL, right and left; RN, right coronary and non-coronary.

\section{Strengths and limitations}

To our knowledge, this is the largest prospective multicentre study to analyse the association of BAV morphology, valvular dysfunction and aorta dilation in a core lab. Patients were referred for a control echocardiographic study in a stable situation and without a previous surgical indication. Therefore, the present study is exposed to the intrinsic referral bias that limits every study based on outpatient data. This was not a community study, and children with BAV were excluded. Thus, a small subset of patients with severely deformed bicuspid valves who had required surgery in childhood or adolescence was not included. Given its cross-sectional nature, this study did not attempt to assess the impact of valve morphotype on aortic disease progression. Patients with aortic coarctation were also excluded since the association of this abnormality generates a specific pattern in ascending aorta dilation. Finally, this study did not include genetic or serum markers, histology or proteomic information. Some factors not included in the study may aid understanding of BAV heterogeneity in valvular dysfunction and aorta dilation.

\section{CONCLUSIONS}

The development of aortic valve dysfunction and aorta dilation in patients with BAV results from complex interactions among 


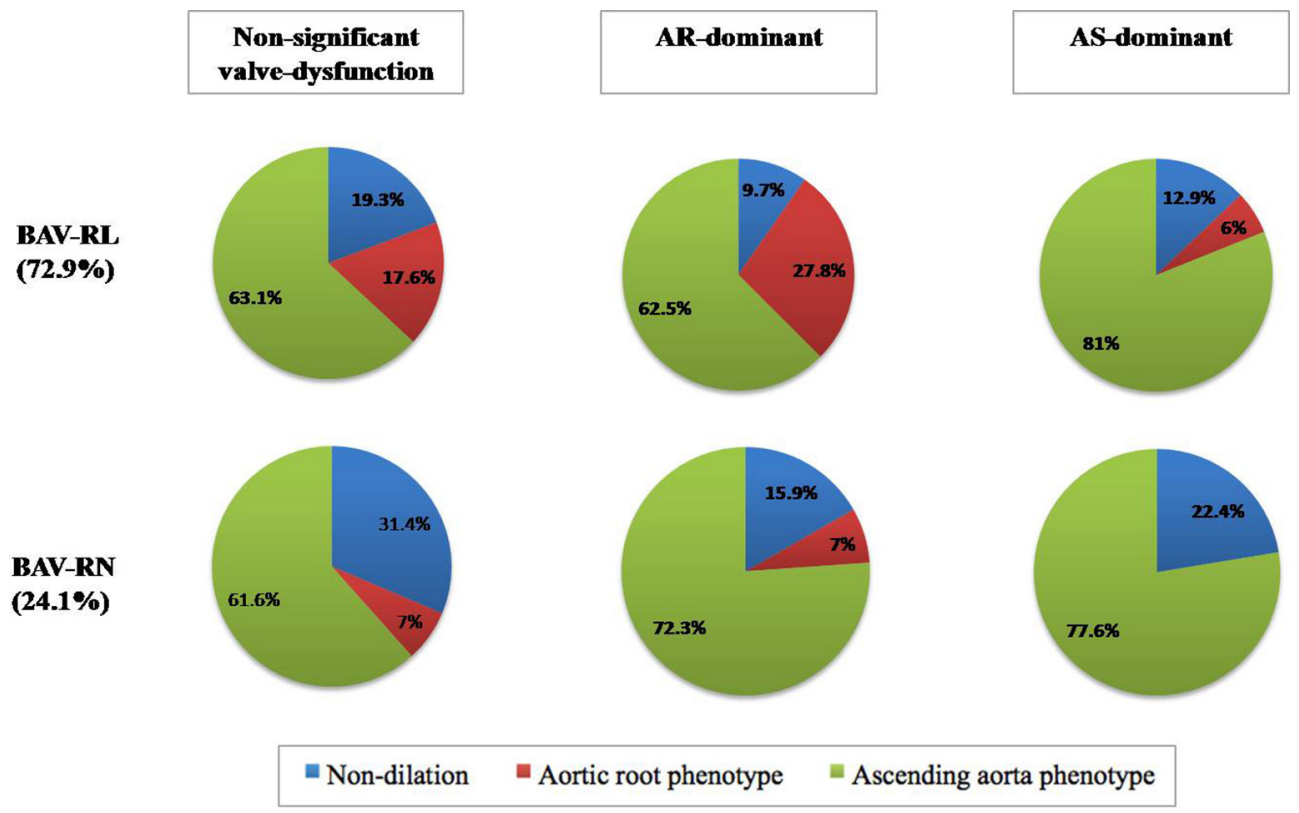

Figure 3 Aorta dilation and phenotype according to valve morphotype and valve dysfunction. Note that non-dilated aorta was more frequent in BAV-RN, aortic root dilation was infrequent in BAV-RN and absent in the presence of AS, and ascending aorta dilation was similar in both BAV types. BAV, bicuspid aortic valve; RL, right and left; RN, right coronary and non-coronary.

valve morphotypes, cardiovascular risk factors and specific haemodynamic conditions. Normofunctional valves are more prevalent in BAV without raphe. AS is associated with valve

\section{Key messages}

\section{What is already known on this subject?}

Bicuspid aortic valve (BAV) is the most common congenital heart abnormality associated with early valvular dysfunction and ascending aorta dilation with high heterogeneity. Aortic valve and/or ascending aorta surgery is required in around 25\%-30\% of adult patients 20 years after BAV diagnosis.

\section{What might this study add?}

This study showed that aortic stenosis was more frequent in BAV-right-non-coronary (RN) and related to the presence of raphe, age and cardiovascular risk factors, while aortic regurgitation was related to male sex and valve prolapse. Aortic root was dilated in $34 \%$ of cases and ascending aorta in $76 \%$. Aortic root dilation phenotype was associated with younger age, male sex and aortic regurgitation, and less frequent in BAV-RN, while ascending aorta phenotype was similar among valvular morphotypes and slightly less frequent in normofunctional valve $(67 \%)$. Aortic arch is more dilated in BAV-RN and BAV-LN than BAV-RL.

\section{How might this impact on clinical practice?}

Despite significant BAV heterogeneity, several associations among valve morphotypes, cardiovascular risk factors and haemodynamic conditions may help to stratify the risk of valvular dysfunction and aorta dilation. Control of cardiovascular risk factors and development of new therapeutic strategies may prevent the progression of valve calcification and aortic stenosis, mainly in patients with BAV-RN. Futhermore, the aorta dilation pattern is related to valve morphotype and dysfunction. These associations may help in choosing the best multimodality imaging follow-up test and for surgical treatment strategies. calcification and more frequently with BAV-RN and the presence of cardiovascular risk factors, whereas AR is more frequent in men and associated with sigmoid prolapse. Therefore, particularly in patients with BAV-RN and raphe, the control of cardiovascular risk factors should be optimised. Although ascending aorta is the most commonly dilated aorta segment, aortic root dilation is present in one-third of patients and associated with $\mathrm{AR}$. In contrast to BAV-RL, BAV-RN spares aortic root dilation but enlarges the ascending aorta more distally, including the aortic arch. Therefore, periodic follow-up by MRI or CT is particularly indicated in this BAV morphotype.

Despite these significant associations, further studies are required to identify new imaging haemokinetic parameters, biomarkers and genetic factors that may provide an individualised stratification of the risk for valvular dysfunction and aorta dilation. Control of cardiovascular risk factors and new drug treatments might reduce valvular degeneration progression and aortic dilation.

\section{Author affiliations}

'Servei de Cardiologia, Hospital Universitari Vall d'Hebron, CIBER-CV, Barcelona, Spain

${ }^{2}$ Servicio de Cardiología, Hospital Universitario Virgen Macarena, CIBER-CV, Sevilla, Spain

${ }^{3}$ Servicio de Cardioloxía, Complexo Hospitalario Universitario de Vigo, Vigo, Spain ${ }^{4}$ Servicio de Cardiología, Hospital Gregorio Marañón, CIBER-CV, Madrid, Spain ${ }^{5}$ Unidad de Gestión Clínica del Corazón, Hospital Universitario Virgen de la Victoria de Málaga, CIBER-CV, Málaga, Spain

${ }^{6}$ Servicio de Cardiología, Hospital Universitario Doce de Octubre, CIBER-CV, Madrid, Spain

${ }^{7}$ Servicio de Cardiología, Hospital Clínico Universitario Virgen de la Arrixaca, IMIB-

Arrixaca, CIBER-CV, Murcia, Spain

${ }^{8}$ Servicio de Cardiologia (ICICOR), Hospital Clínico Universitario, CIBER-CV,

Valladolid, Spain

Acknowledgements The authors would like to thank Christine O'Hara for the help with the English language of the manuscript.

Collaborators Hospital Universitari Vall d'Hebron, Barcelona: Laura Gutiérrez, Ruben Fernández and Teresa González-Alujas. Hospital Universitario Virgen Macarena, Sevilla: Irene Mendez; Complexo Hospitalario Universitario de Vigo: Mireya Castro Verdes and Cristina Victoria Iglesia Carreño. Hospital Gregorio Marañón, Madrid: 
María Eugenia Vázquez and Ana González-Mansill. Hospital Universitario Virgen de la Victoria de Málaga: Isabel Rodríguez-Bailón. Hospital Universitario Doce de Octubre, CIBER cardiovascular, Madrid: Sagrario Fernández Casares and Carmen Jiménez López-Guarch. Hospital Clínico Universitario Virgen de la Arrixaca, Murcia: Gonzalo de la Morena and M. José Oliva. Hospital Clínico Universitario, Valladolid: Teresa Sevilla.

Contributors AE planned, conducted the study and wrote the manuscript. PG, FC-I, JB, JR-C, VS, DS, RA and AC collected and acquired echocardiographic studies. GM included information in database. AS-A and JR-P contributed in statistical analysis. GT and LG, expert echocardiographers, analysed and measured all studies. DG-D helped in writing the final version of the manuscript.

Funding Supported by grants from the Fondo de Investigación Sanitaria, Red de Investigación Cooperativa de las Enfermedades Cardiovasculares, CIBER-CV Instituto de Salud Carlos III, Ministerio de Sanidad y Consumo and cofunded by European Union (ERDF/ESF) FIS-PI11/01081.

Competing interests None declared.

Patient consent Obtained.

Ethics approval CEIC Hospital Universitari Vall d'Hebron.

Provenance and peer review Not commissioned; externally peer reviewed.

(c) Article author(s) (or their employer(s) unless otherwise stated in the text of the article) 2018. All rights reserved. No commercial use is permitted unless otherwise expressly granted.

\section{REFERENCES}

1 Verma S, Siu SC. Aortic dilatation in patients with bicuspid aortic valve. N Eng/ J Med 2014;370:1920-9

2 Braverman AC. Aortic involvement in patients with a bicuspid aortic valve. Heart 2011;97:506-13.

3 Siu SC, Silversides CK. Bicuspid aortic valve disease. J Am Coll Cardiol 2010;55:2789-800.

4 Michelena HI, Prakash SK, Della Corte A, et al. Bicuspid aortic valve: identifying knowledge gaps and rising to the challenge from the International Bicuspid aortic valve Consortium (BAVCon). Circulation 2014;129:2691-704.

5 Hahn RT, Roman MJ, Mogtader AH, et al. Association of aortic dilation with regurgitant, stenotic and functionally normal bicuspid aortic valves. J Am Coll Cardiol 1992;19:283-8.

6 Della Corte A, Bancone C, Quarto C, et al. Predictors of ascending aortic dilatation with bicuspid aortic valve: a wide spectrum of disease expression. Eur I Cardiothorac Surg 2007;31:397-405.

7 Michelena HI, Desjardins VA, Avierinos JF, et al. Natural history of asymptomatic patients with normally functioning or minimally dysfunctional bicuspid aortic valve in the community. Circulation 2008;117:2776-84.

8 Tzemos N, Therrien J, Yip J, et al. Outcomes in adults with bicuspid aortic valves. JAMA 2008:300:1317-25

9 Beppu S, Suzuki S, Matsuda H, et al. Rapidity of progression of aortic stenosis in patients with congenital bicuspid aortic valves. Am J Cardiol 1993;71:322-7.

10 Schaefer BM, Lewin MB, Stout KK, et al. The bicuspid aortic valve: an integrated phenotypic classification of leaflet morphology and aortic root shape. Heart 2008:94:1634-8
11 Della Corte A, Bancone C, Buonocore M, et al. Pattern of ascending aortic dimensions predicts the growth rate of the aorta in patients with bicuspid aortic valve. JACC Cardiovasc Imaging 2013;6:1301-10.

12 Khoo C, Cheung C, Jue J. Patterns of aortic dilatation in bicuspid aortic valveassociated aortopathy. J Am Soc Echocardiogr 2013;26:600-5.

13 Kang J-W, Song HG, Yang DH, et al. Association between bicuspid aortic valve phenotype and patterns of valvular dysfunction and bicuspid aortopathy. JACC Cardiovasc Imaging 2013;6:150-61.

14 Kim YG, Sun BJ, Park GM, et al. Aortopathy and bicuspid aortic valve: haemodynamic burden is main contributor to aortic dilatation. Heart 2012;98:1822-7

15 Yousry M, Rickenlund A, Petrini J, et al. Real-time imaging required for optimal echocardiographic assessment of aortic valve calcification. Clin Physiol Funct Imaging 2012;32:470-5.

16 Campens L, Demulier L, De Groote K, et al. Reference values for echocardiographic assessment of the diameter of the aortic root and ascending aorta spanning all age categories. Am J Cardiol 2014;114:914-20.

17 Della Corte A, Bancone C, Dialetto G, et al. The ascending aorta with bicuspid aortic valve: a phenotypic classification with potential prognostic significance. Eur $J$ Cardiothorac Surg 2014:46:240-7.

18 Taylor AP, Yadlapati A, Andrei AC, et al. Statin use and aneurysm risk in patients with bicuspid aortic valve disease. Clin Cardiol 2016;39:41-7.

19 Michelena HI, Suri RM, Katan O, et al. Sex differences and survival in adults with bicuspid aortic valves: verification in 3 contemporary echocardiographic cohorts. J Am Heart Assoc 2016;5:e004211.

20 Sabet HY, Edwards WD, Tazelaar HD, et al. Congenitally bicuspid aortic valves: a surgical pathology study of 542 cases (1991 through 1996) and a literature review of 2,715 additional cases. Mayo Clin Proc 1999;74:14-26.

21 Kong WK, Delgado V, Poh KK, et al. Prognostic implications of raphe in bicuspid aortic valve anatomy. JAMA Cardiol 2017;2:285.

22 Girdauskas E, Rouman M, Disha K, et al. Morphologic and functional markers of Aortopathy in Patients with bicuspid aortic valve insufficiency Versus Stenosis. Ann Thorac Surg 2017;103:49-57.

23 Girdauskas E, Rouman M, Disha K, et al. Aortic dissection after previous aortic valve replacement for bicuspid aortic valve disease. J Am Coll Cardiol 2015;66:1409-11.

24 Nistri S, Sorbo MD, Marin M, et al. Aortic root dilatation in young men with normally functioning bicuspid aortic valves. Heart 1999;82:19-22.

25 Robicsek F, Thubrikar MJ, Cook JW, Cook M.,., et al. The congenitally bicuspid aortic valve: how does it function? why does it fail? Ann Thorac Surg 2004;77:177-85.

26 Girdauskas E, Rouman M, Disha K, et al. Functional aortic root parameters and expression of aortopathy in bicuspid versus tricuspid aortic valve stenosis. J Am Coll Cardiol 2016:67:1786-96.

27 Bissell MM, Hess AT, Biasiolli L, et al. Aortic dilation in bicuspid aortic valve disease: flow pattern is a Major contributor and differs with valve fusion type. Circ Cardiovasc Imaging 2013;6:499-507.

28 Mahadevia R, Barker AJ, Schnell S, et al. Bicuspid aortic cusp fusion morphology alters aortic three-dimensional outflow patterns, wall shear stress, and expression of aortopathy. Circulation 2014;129:673-82.

29 Sievers HH, Stierle U, Hachmann RM, et al. New insights in the association between bicuspid aortic valve phenotype, aortic configuration and valve haemodynamics. Eur $J$ Cardiothorac Surg 2016;49:439-46. 